\title{
SERO-EPIDEMIOLOGY OF TOXOPLASMOSIS AMONGST PREGNANT WOMEN IN THE GREATER ACCRA REGION OF GHANA
}

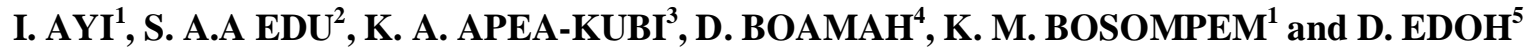 \\ ${ }^{1}$ Parasitology Department, Noguchi Memorial Institute for Medical Research,College of Health Sciences, \\ University of Ghana, Box LG 581, Legon, Accra, Ghana; ${ }^{2}$ Council for Scientific and Industrial Research, \\ Animal Research Institute, P. O. Box AH 20, Achimota, Accra, Ghana; ${ }^{3}$ Obstetrics \& Gynaecology Depart- \\ ment, Korle-Bu Teaching Hospital, Accra, Ghana. ${ }^{4}$ Immunogenetics Department, Institute of Tropical Medi- \\ cine (NEKKEN), Nagasaki University, 1-12-4 Sakamoto, Nagasaki City, 852-8523, Japan and ${ }^{5}$ Zoology De- \\ partment, University of Ghana, Legon, Accra, Ghana
}

\author{
Author for correspondence: Dr. Irene Ayi \\ Conflict of interest: None declared
}

\section{SUMMARY}

Objectives: To investigate Toxoplasma infection among pregnant women in relation to exposure to infection risk, age and pregnancy-related risk factors.

Design and Methods: This cross-sectional study involved 294 pregnant women attending ante-natal clinic in Accra who consented to participate. Personal and Toxoplasma infection risk related data were obtained by questionnaire interviews. Venous blood was safely drawn from each participant and spun to obtain sera. Each of the 159 randomly selected serum samples was tested for specific anti-Toxoplasma (anti-T. gondii) antibodies $\operatorname{IgG}, \operatorname{IgA}$ and $\operatorname{IgM}$ using a commercial ELISA kit (Calbiotech Inc., CA). ELISA results were correlated with exposure to possible infection risk factors as well as age and pregnancy-related risk factors.

Results: The 159 women aged 15-40 years in their first, second and third trimesters, numbered 29, 70 and 60 , respectively. An overall anti- $T$. gondii antibodies $\mathrm{IgG}, \operatorname{IgA}$ and IgM seroprevalence of $92.5 \%$ (147/159) was recorded, with $4.1 \%(6 / 147)$ of them having antiIgG only. The remaining $88.7 \%(141 / 159)$ had antiToxoplasma antibodies $\operatorname{IgG}, \operatorname{IgA}$ and $\operatorname{IgM}$ in various combinations and consisted of $17.7 \%(25 / 141)$ in their first, $44.0 \%(62 / 141)$ in their second, and $38.3 \%$ $(54 / 141)$ in their third, trimesters. Twelve women (7.6\%) were seronegative for all 3 antibodies

Conclusions: Seroprevalence was high among the women and exposure to contact with cats' faeces was found to be the major $T$. gondii infection risk factor. Age and pregnancy-related risk factors did not have association with $T$. gondii infection within the limitations of this study.

Keywords: Toxoplasma, pregnancy, infection risk, seroprevalence, Ghana

\section{Email:ayi@noguchi.mimcom.org}

\section{INTRODUCTION}

Toxoplasmosis is a zoonotic disease caused by the protozoan parasite, Toxoplasma gondii. About $20 \%$ to $90 \%$ of the world's adult population in different regions are reported to have had contact with the parasite. ${ }^{1}$ Toxoplasma infection may be acquired or congenital and the disease is important for its serious implications in immuno-suppressed individuals including pregnant women as well as its severe consequences on foetuses in congenital transmission. The rate of transmission to the foetus is $10-15 \%$ in the first trimester of gestation, which may increase to $68 \%$ in the third trimester. ${ }^{2}$ Thus maternal infections early in pregnancy are less likely to be transmitted to the foetus than infections later in pregnancy, but early foetal infections are likely to have more severe consequences than late infections. ${ }^{3}$

Although most infected foetuses (approximately 75\%) do not have obvious clinical signs at birth, many (approximately $80-85 \%$ ) are likely to have manifestations such as chorioretinitis and mental retardation later in life. ${ }^{4,5}$ Infection during the first trimester may lead to spontaneous abortion, stillbirth, or overt disease in the neonate while infection acquired later during pregnancy is usually asymptomatic in the neonate, and may not be recognized. ${ }^{6}$ Maternal toxoplasmosis as a risk factor for spontaneous abortion was investigated and infection was found much more frequently in women with habitual abortion (18.5\%) than in the normal pregnancy group $(5.9 \%){ }^{7}$

Toxoplasma oocyst shed in cat (the definitive host) faeces sporulate in the soil under warm, humid conditions and could contaminate water and food, especially fruits and vegetables. 
Most cases of toxoplasmosis in humans are probably acquired by ingestion of sporulated oocyst in soil or contaminated unwashed/unpeeled vegetables and fruits; tachyzoite in unpasteurised milk and cysts in raw or undercooked infected meat. Although beef is a potential source of infection, pork and lamb are known to be the most common sources of contamination. Filth flies (that is, flies that develop on animal dung, carcasses, faeces, blood or garbage) such as the common housefly, Musca domestica Linnaeus and the oriental blow fly, Chrysomya megacephala (Fabricius) also have the capability of carrying viable Toxoplasma oocyst (for 1 to 2 days) from cat faeces to food. ${ }^{8}$ Needlestick injuries, cuts, blood transfusion and organ transplantation have also been mentioned as possible infection risk factors. ${ }^{9}$ Infection with Toxoplasma depends on the level and frequency of exposure to such factors as mentioned above and therefore usually affects scattered individuals clinically.

There have however been a few reported cases of outbreaks in some areas implicating drinking unfiltered water with possible oocyst contamination. ${ }^{10,11}$ Outbreaks involving a group of students of the Cornel University Medical College implicating eating of undercooked hamburgers and, in Sao Paulo 110 persons admitting to eating undercooked meat after being diagnosed with toxoplasmosis have been reported. ${ }^{12,13}$

Toxoplasmosis may be diagnosed by direct and indirect methods. Direct methods include Polymerase Chain Reaction (PCR) for the detection of $T$. gondii DNA from samples of body fluids, demonstration of the organism through mouse inoculation, cell culture, ophthalmic testing and radiological studies. ${ }^{9,14}$ Indirect diagnosis is mainly serological methods for the detection of anti- $T$. gondii antibodies. The most commonly used serologic tests to detect the presence of anti- $T$ gondii IgG and IgM antibodies are the Sabin-Feldman dye test, indirect fluorescent antibody (IFA), and agglutination tests, or ELISA. ${ }^{15,16}$

In antibody detection for diagnosis of $T$. gondii infection, anti-IgM and/or $\operatorname{IgA}$ antibodies in combination with $\mathrm{IgG}$ is reported to indicate mostly recent or acute infections because these antibodies are not usually in acquired immunity and very rare in chronic infections. Furthermore, $\operatorname{Ig} \mathrm{A}$ antibodies persist for over 3 or 4 months following acquired $T$. gondii infections and low titres of $\operatorname{IgG}$ antibodies are usually in patients with active toxoplasmic chorioretinitis, in which IgM antibody cannot usually be detected. ${ }^{17}$

In Ghana, studies on toxoplasmosis in the past had employed mostly anti-Toxoplasma $\mathrm{IgG}$ detection in patients' sera. Seroprevalence studies for toxoplasmosis in farm animals indicated seropositivity of $39 \%$ in pigs, $26.8 \%$ in goats and $33.2 \%$ in sheep. ${ }^{18,19}$ Toxoplasma antigens had also been detected in urine from $87.0 \%$ of Toxoplasma infected eye patients and $73.1 \%$ pregnant women, screened. ${ }^{20}$

A study published in 1997, suggests that Toxoplasma antibodies are more prevalent in women having cats at home than in women who do not possess cats. ${ }^{7}$ The most common mode of Toxoplasma infection transmission has not been established in Ghana. This study sought to determine the Toxoplasma infection status of pregnant women at different gestation periods and its association with exposure to possible infection risk factors.

\section{METHODS}

This cross-sectional study was reviewed and approved by the Scientific and Technical Committee and ethical clearance was obtained from the Institutional Review Board of the NMIMR, UG. Permission was also obtained from appropriate hospital authorities. Study participants were recruited based on written informed consent after explanation of the study. Closed-ended questionnaires were administered to collect personal information as well as Toxoplasma infection-related data. Venous blood samples were collected to obtain sera for detection of anti-Toxoplasma antibodies (IgG, IgA and IgM) by commercial ELISA kits. Case-patients were defined as pregnant women with positive test results for any of the 3 anti-Toxoplasma $\operatorname{IgG}, \operatorname{IgA}$ or $\operatorname{IgM}$, or combinations of the 3 antibodies. Associations between responses to relevant questions on exposure to infection risk factors and infection status were determined by level of statistical significance using Chi Square analysis.

The study population comprised of volunteer pregnant women aged 15-42 years attending antenatal clinics from February to May 2006 at the Korle-Bu Teaching and Achimota Hospitals in Accra, Ghana. Study participants were recruited based on written consent forms after educating them on the disease and study details. Korle-Bu Teaching Hospital (KBTH) being the main referral and teaching hospital provides health services for the entire country as well as some neighbouring countries whiles Achimota Hospital is a community hospital providing health services for several communities in the $\mathrm{Ga}$ and neighbouring Districts.

Questionnaires were administered by interview in participants' language for some whiles others selfadministered with the appropriate guidance. Questions sought personal information on participants including their age, number of previous pregnancies, any past occurrence of still births and/or spontaneous abortions, 
gestational period of current pregnancy as well as their knowledge about the disease and exposure to possible Toxoplasma infection transmission risk factors. The possible infection transmission risk factors considered in the questionnaire included handling and eating of meat from high transmission risk animals (pigs, sheep and goats) and contact with cat and cat sandboxes. Concerning meat, they were asked how often they handled and/or ate the meat on daily or weekly basis, in what form they preferred their meat (thoroughly and/or partially cooked), and the meat they preferred for khebab (pieces of meat stringed on skewers and charcoalgrilled, usually prepared and sold outside the home). The women were also asked if they owned and/or had cats in their house or immediate surroundings. Those that indicated owning cats were asked if they had sandboxes for them.

A previous study had estimated the Toxoplasmosis prevalence to be $89 \%$ among eye patients. (Prevalence among eye patients was used because toxoplasmic eye lesions in adults are a consequence of congenital toxoplasmic infection of the foetus). ${ }^{21}$ To achieve a $95 \%$ confidence interval of width $\pm 5 \%$, a minimum of 150 serum samples were necessary for analysis. For the questionnaire study in an attempt to determine the most common mode of infection, no previous data was found. Assuming a $20 \%$ chance of infection by any applicable mode, a minimum of 245 individuals were needed for the questionnaire survey to achieve a $95 \%$ confidence interval of width $\pm 5 \%$.

\section{Antibody testing}

Information, including personal and responses to questionnaire interviews, on all participants was computerised using MS Excel spreadsheet and some were selected by the simple random sampling method of computer generated random numbers. Serum samples of the selected participants were tested for anti-T. gondii antibodies using ELISA.

Between 2 and $3 \mathrm{ml}$ of venous blood was obtained from each participant using a sterile disposable hypodermal syringe fitted with a 23 gauge needle and dispensed from the syringe barrel into a sterile tube and allowed to clot. Clots were removed and discarded and the sample tubes span at $500 \times \mathrm{g}$ to precipitate RBCs. Clear sera were carefully collected, aliquot into Eppendorf tubes and stored frozen at $-20^{\circ} \mathrm{C}$ until tested. Each sample was tested for the presence of anti-Toxoplasma antibodies, $\operatorname{IgG}, \operatorname{IgM}$ and $\operatorname{IgA}$, using commercial ELISA Kit (Cal biotech Inc., CA) and following manufacturer's instructions. ELISA results were recorded using a micro-plate reader (Multiskan Ascent v1.24), as a measure of optical densities (OD) of the reaction intensity of $T$. gondii antigen and serum anti-T. gondii antibodies at a filter wavelength of $414 \mathrm{~nm}$. Cut-off points and antibody index calculations were done according to manufacturers' recommendation to categorise seropositive (antibody index >1.1), borderline positive (antibody index 0.9 to 1.1 ) and seronegative (antibody index <0.9) samples. In this study however, all serum samples with intensity of antibody index 0.9 to 1.1 (borderline positives) were classified as indeterminate and considered as seronegative pending test repeat and when necessary re-tested using the IFA test.

\section{Statistical Analysis}

The seroprevalence of anti- Toxoplasma antibodies was estimated for the various immunoglobulins tested. Data were analysed with univariate analysis to determine risk factors considered to be of biological importance $(\mathrm{p} \leq 0.05)$ for association with $T$. gondii infection. Prevalence odds ratio (POR) was used to estimate the possible association between infection transmission and the risk factors. Statistical significance was determined by Chi-square tests. Analysis was performed using SPSS.

\section{RESULTS}

A total of 294 pregnant women aged 15-42 years (mean age: 28.06 years; std. dev.: 5.528 years) volunteered to participate in the study after informed consent. Two pregnant women $(0.7 \%)$ could not give their ages. The 25-29 years age group recorded the highest participation, $37.1 \%(109 / 294)$ and the $40-44$ year-olds had the least of $2.0 \%(6 / 294)$. All the 294 pregnant women responded to over $90 \%$ of question items in a questionnaire interview, however, not all of them volunteered to give blood for testing.

About $10.2 \%(30 / 294)$ of the pregnant women were in their first trimester with $50.7 \%(149 / 294)$ and $38.4 \%$ $(113 / 294)$ in the second and third trimesters, respectively, while $0.7 \%(2 / 294)$ could not give the age of their pregnancy. Only one woman had some knowledge about Toxoplasmosis because she had been tested for it in the past.

Serum samples from 159 of those who offered blood were selected for anti- $T$. gondii antibody testing. The 159 pregnant women aged 15-40 years (mean age: 28.22 years; std. Dev. 5.143 years) (Figure 1) were grouped into trimester gestational periods (Figure 2).

The 159 pregnant women sero-tested consisted of 8 $5.0 \%$ aged $15-19$ years, $16.4 \% 20$ to 24 years old, $41.5 \%$ within $25-29$ years, $25.2 \%$ in the $30-34$ years age group, $9.4 \%$ and $1.3 \%$ in the $35-39$ and $40-44$ years age groups, respectively. For gestational periods, $18.2 \%$ were in their first trimester, $44.0 \%$ in the second and $37.7 \%$ in the third trimester. Forty-four $(27.7 \%)$ of 
the pregnant women were primigravida (had no previous pregnancies). In all 110 (69.2\%) of them (including the 44 primigravida) had no history of spontaneous abortion or still-birth, $37(23.3 \%)$ had one, $11(6.9 \%)$ had two and $1(0.6 \%)$ had 3 or more (Table 1$)$. Parity of participants was deduced from their responses to number of past pregnancies and past experiences of spontaneous abortions and stillbirths. The 44 primigravida were obviously nulliparous. The remaining 66 with no past experience of spontaneous abortions or stillbirths could be grouped depending on the number of previous deliveries as Parity 1 to Parity $>3$ except one of them that did not indicate the number of past pregnancies.

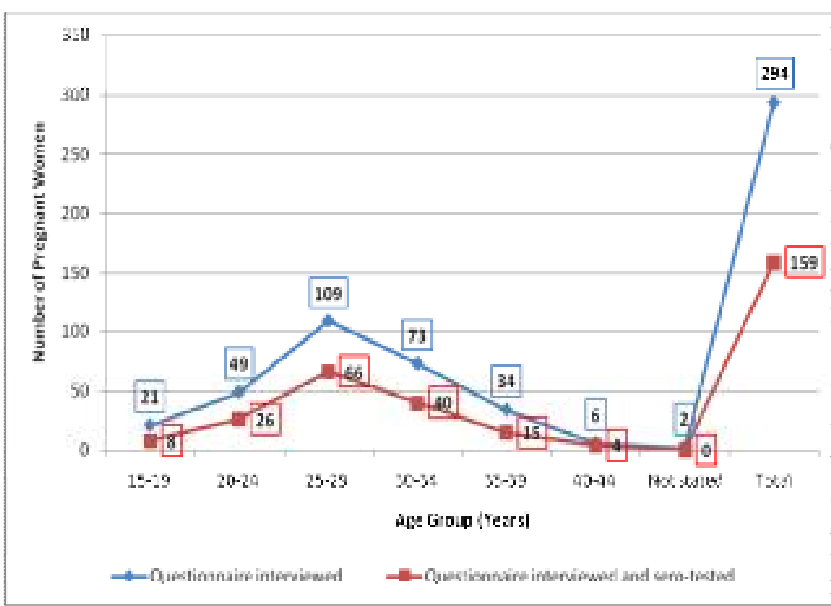

Figure 1 Age distribution of pregnant women involved in study

Table 1 Number of past pregnancies in relation to past experiences of spontaneous abortions and stillbirths among the pregnant women tested for anti-Toxoplasma gondii antibodies

\begin{tabular}{|c|c|c|c|c|c|}
\hline \multirow[t]{3}{*}{ Past pregnancies } & \multicolumn{4}{|c|}{$\begin{array}{c}\text { No. of women responding to past experience(s) } \\
\text { of stillbirths/spontaneous } \\
\text { Abortions/miscarriages } \\
(\%) \\
\end{array}$} & \multirow[t]{3}{*}{ Total (\%) } \\
\hline & \multirow[b]{2}{*}{ No } & \multicolumn{3}{|c|}{ Yes } & \\
\hline & & Once & Twice & Thrice & \\
\hline None & $44(27.7)$ & 0 & 0 & 0 & $44(27.7)$ \\
\hline One & $29(18.2)$ & $11(6.9)$ & 0 & 0 & $40(25.2)$ \\
\hline Two & $15(9.4)$ & $16(10.1)$ & $5(3.2)$ & 0 & $36(22.6)$ \\
\hline Three & $16(10.1)$ & $8(5.0)$ & $4(2.5)$ & 0 & $28(17.6)$ \\
\hline More than three & $5(3.2)$ & $2(1.3)$ & $2(1.3)$ & $1(0.6)$ & $10(6.3)$ \\
\hline \multirow[t]{2}{*}{ Not answered } & $1(0.6)$ & 0 & 0 & 0 & $1(0.6)$ \\
\hline & $110(69.2 \%)$ & $37(23.3)$ & $11(6.9)$ & $1(0.6)$ & $159(100.0)$ \\
\hline
\end{tabular}

\section{ELISA results}

The ELISA results illustrated the relative concentration of circulating anti-T. gondii antibodies in the seroposi-

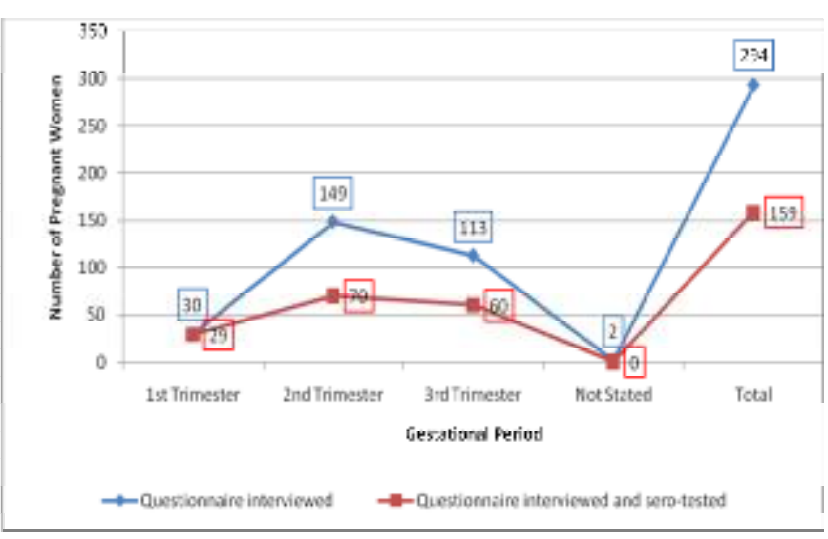

Figure 2 Distribution of pregnant women involved in study by gestational period

The other 49 women with one, two or three past spontaneous abortions and/or stillbirths could be assigned Parity 0 to Parity $\geq 3$ depending on the number of past pregnancies. Only 1 of the participants (a 36 year-old in her second trimester) had knowledge of toxoplasmosis and had previously been tested for the infection with a negative result. This woman, according to questionnaire response had had contact with cat sandbox but did not own or have a cat in her surroundings whiles she preferred beef only for consumption at home or as 'khebab'. The current test of her serum resulted as positive for anti-Toxoplasma $\mathrm{IgG}$ and $\operatorname{IgM}$. 
lowest intensity (1.2 to 2.0) involved 64 women for $\operatorname{IgM}, 17$ for IgG and 10 for IgA. Antibody index of 2.1 to 3.5 was found in 111 women ( 58 for $\mathrm{IgG}, 42$ for $\operatorname{IgM}$ and 11 for $\operatorname{IgA}$ ), whiles 3.6 to 5.0 was found in 112 women (40 for $\operatorname{IgG}, 14$ for $\operatorname{IgM}$ and 58 for $\operatorname{IgA}$ ).

An overall prevalence of $92.5 \%$ (147/159) serum antiToxoplasma IgG (73.6\%), IgA (64.8\%) and IgM (76.1\%) was estimated by ELISA. A combination of all 3 antibodies $(\operatorname{IgG}+\operatorname{Ig} \mathrm{A}+\operatorname{IgM})$ was detected in 80 (54.4\%) of the 147 seropositive samples, $\operatorname{IgG}+\operatorname{IgM}$ in $13(8.8 \%), \operatorname{IgG}+\operatorname{IgA}$ in $18(12.2 \%), \operatorname{IgM}+\operatorname{IgA}$ in 3 (2.0\%), IgM only in $25(17.0 \%)$, IgA only in $2(1.4 \%)$ and IgG only in $6(4.1 \%)$. The remaining $12(7.5 \%)$ of the 159 pregnant women were considered seronegative for all the antibodies, to be re-tested for confirmation. Three (3) out of the 12 were clearly negative (antibody index <0.9) for all the 3 antibodies whiles the other 9 had borderline antibody indices $(0.9$ to 1.1$)$ for the respective antibodies detected in the proportion of $6 \operatorname{IgM}$ only, 2 IgG only and $1 \mathrm{IgG}+\mathrm{IgM}$.

Overall seroprevalence amongst the first trimesters was $17.0 \%(27 / 159), 40.9 \%(65 / 159)$ in second trimesters and $34.6 \%(55 / 159)$ in third trimesters. Seroprevalence of Toxoplasma infection among the women within the different gestation periods were $93.1 \%$ (27/29) for first, $92.9 \%(65 / 70)$ for second and $91.7 \%(55 / 60)$ for third trimesters. A very high prevalence was observed for all trimesters and there was no significant difference $(p>0.05)$ in the prevalence values. Overall seroprevalence as categorized by age groups, in years, was as follows: $15-20,5.0 \%$; 21-25, 24.5\%; 26-30, 35.9\%; 31$35,18.2 \%$; and $36-40,8.8 \%$. Seroprevalence was generally high (88.9-97.0\%) within all the age groups with no statistical significance $(p>0.05)$. Within high infection risk occupation group (caterer, fruit and vegetable vendors, traders in fresh meat) seroprevalence was $86.7 \%(13 / 15)$ and $90.6 \%$ (126/139) within low risk occupation group (hair dressers, office workers, teachers, students and others). Over $90.0 \%$ of the women were seropositive irrespective of whether their responses to possible infection transmission risk factors were affirmative or otherwise.

In the univariate analysis of the Toxoplasma infection status of the pregnant women and the related risk factors almost all the factors considered were found not to be associated with risk of infection. However, high likelihood of exposure to cats' faeces by owning cat(s) and/or having cats in your immediate surrounding and/or handling cats' sandboxes was possibly associated with the risk of $T$. gondii infection (OR 6.20, 95\% Confidence Interval [CI] 0.78-49.38, $\mathrm{p} \leq 0.05)$.
Between $91-100 \%$ of the women without, or with one or more past experiences of spontaneous abortions and/or still birth tested seropositive for anti- $T$. gondii antibodies, whiles $87-94 \%$ of them responded that they had contact with cat faeces through sandbox. One woman who indicated to have had three or more abortions and/or stillbirth in the past tested positive for all 3 anti- $T$. gondii antibodies, responded 'Yes' to owning a cat and was in the high infection risk occupation group.

\section{DISCUSSION}

This study aimed at investigating $T$. gondii infection among pregnant women in relation to exposure to infection risk, age and pregnancy-related risk factors. An overall anti- $T$. gondii antibodies seroprevalence of $92.5 \%(147 / 159)$ was recorded among the pregnant women from the two hospitals which is relatively higher than previously recorded values in other studies. $^{20}$

In that study the Latex Agglutination Test (which detects anti- $T$. gondii IgG only was employed and could therefore not differentiate between recent and past infection. The current study although detected other antibodies to identify past and recently infected individuals it would have been most appropriate to have confirmed the infection status of the individuals by using another comparable method such as IFA as the "Gold Standard". The study could also have investigated if the pregnant women handled freshly slaughtered raw meat and if they did so with broken skin on their hands or not to assess it as a route of infection.

In the current study, combinations of specific antiToxoplasma antibodies $\mathrm{IgG}, \mathrm{IgA}$ and $\mathrm{IgM}$ was detected in $95.9 \%(141 / 147)$ of the pregnant women which is comparable with a previous $81 \%(\mathrm{p}>0.05)$ prevalence in a circulatory $T$. gondii antigens detection study to diagnose on-going or recent infections. ${ }^{21}$ In antibody detection for diagnosis of Toxoplasma infection, anti$\operatorname{IgM}$ and/or IgA antibodies in combination with $\operatorname{IgG}$ suggests mostly recent, acute or ongoing infections as has been reported that, $\operatorname{IgA}$ antibodies associated with IgM antibodies indicate acute infections because these antibodies are not usually in acquired immunity and very rare in chronic infections. ${ }^{17}$ Furthermore, the presence of $\operatorname{IgG}$ together with $\operatorname{IgA}$ in circulation indicates recent acquired infection because, IgA antibodies persist for over 3 or 4 months following acquired Toxoplasma infections and low titres of $\mathrm{IgG}$ antibodies are usually in patients with active toxoplasmic chorioretinitis, in which IgM antibody cannot usually be detected. ${ }^{17}$

The combinations of anti-IgG, IgA and IgM antibodies detected is an indication that most of the pregnant 
women (141) had acquired the infection for the first time because only 6 out of the 147 seropositive had $\mathrm{IgG}$ only. Presence of anti-IgM and/or IgA strongly suggests the presence of parasite antigens in circulation implying possible presence of parasites, in this case, tachyzoites, in circulation as well. Although prepregnancy Toxoplasma infection status of the pregnant women is unknown, it could be deduced from the combination of antibodies detected and age of pregnancy, that $86.2 \%(25 / 29)$ of those in their first trimester could have acquired the infection up to 3 months before conception or during pregnancy. They stand a $10-15 \%$ risk of transmitting the infection congenitally with severe consequences to the foetus. For the second and third trimester pregnant women, 96.7\% (116/120) might have contracted the infection during pregnancy and stand an increasing (up to 68\%) risk of infecting their unborn babies, but with less severe consequences to the infected babies. ${ }^{3}$ This is therefore an indication that, almost all the seropositive pregnant women identified in this study stand a high risk of transmitting the infection to their foetuses. In addition, the lowest positive antibody index of 1.2-2.0 for IgG was observed in 17 pregnant women who could be said to have active toxoplasmic chorioretinitis.

The very high seroprevalence of recent or ongoing infections could be as a result of primary infection or a reactivated past infection (especially, in the pregnant women that tested positive for anti- $T$. gondii IgG only) which is reported to occur in immuno-compromised individuals. However, antibodies, particularly IgM may not rise during reactivation of the encysted form of the parasite. ${ }^{3}$ Detection of IgM and/or IgA in majority of the pregnant women could also be attributed to some physiological changes, stressful demands and the general hormonal imbalances associated with pregnancy that may lower their resistance to diseases. ${ }^{22}$

In this study, we found that the age- and pregnancyrelated risk factors were not significantly associated with $T$. gondii infection. Although frequency of $T$. gondii infection has been reported to increase in older age groups in other studies, the current study showed no apparent association between Toxoplasma infection and age of the pregnant women. ${ }^{23,24,25}$ Previous findings in Ghana reported prevalence of $67.9 \%$ and $82.1 \%$ in pregnant women aged 15-25 and 31-40 years, respectively, whiles this study recorded $29.5 \%$ and $27.0 \%$ for the same age groups. ${ }^{26}$

Although there was no statistically significant association between the handling of meat from high transmission risk animals and Toxoplasma infections, infection through handling of raw meat cannot be ruled out since penetration of vegetative forms (tachyzoites) in mu- cous membranes through broken skin has been reported in some countries. ${ }^{27,28}$ Furthermore, $92.4 \%$ of the pregnant women that responded to handling raw meat were sero-positive and only $4.1 \%$ of them were positive for anti-Toxoplasma IgG alone.

Eating of under-cooked meat from high transmission risk animals and Toxoplasma infections was not found to be associated in this study although, previous studies in Ghana and elsewhere had reported infection in most farm animals especially pigs, sheep and goats. Beef is less likely to be a source of infection. ${ }^{18,19,29,30,31,32}$ Freezing and the thorough cooking of meat significantly reduces the risk of meat-eating-related Toxoplasma infection and could have contributed to the lack of association of Toxoplasma infection. ${ }^{30}$

With the soil-related risk factors, working in a garden with bare hands and also frequently eating unwashed fresh fruits and vegetables showed no significant association with Toxoplasma infection in this study. The non-association of $T$. gondii infection and 'working in a garden with bare hands' could be due to the fact that the study population are urban dwellers and therefore, relatively few (12/159) of them engaged in gardening. However, since $91.7 \%$ (11/12) of them tested positive for anti- $T$. gondii antibodies, this factor could be further investigated in an appropriate setting for a more convincing conclusion.

However, exposure to contact with cats' faeces by means of owning cat(s) and/or having cats in the immediate surrounding and in addition handling cats' sandboxes containing their faeces was found to be associated with Toxoplasma infection. It is possible that most of the cats were infected and excreting oocyst which becomes infective after sporulation in the external environment. A study had suggested that Toxoplasma antibodies were more prevalent in pregnant women with cats at home than in pregnant women that did not possess cats. ${ }^{7}$ To prevent cats from becoming infected, cats must be fed on well cooked meat and if possible, kept indoors to prevent them from hunting or scavenging.

Other notable infection risk factors that could be sources of infection but were not considered in this study could be responsible for the overall high prevalence $(92.5 \%)$ observed. These include the reported capability of filth flies carrying oocyst from cat faeces to food, as well as drinking of contaminated water as observed in some outbreaks. 


\section{CONCLUSION}

The data obtained from this study showed a very high seroprevalence of toxoplasmosis among pregnant women and almost all of them appeared to be recently or acutely infected. The high percentage of $T$. gondii infection among the pregnant women was accompanied by an extremely high level of ignorance about the disease $(99.7 \%)$. Exposure to cats' faeces was identified as the possible $T$. gondii infection risk factor but inadequately confirmed. Further focused studies should be conducted taking into consideration relevant factors such as sample size. The high level of ignorance about the disease may suggest that toxoplasmosis is a neglected disease in Ghana. This therefore calls for 1) intensive education of the populace, especially, women of child-bearing age including pregnant women so preventive measures could be taken; and 2) further initiation of intervention through routine testing of all pregnant women for Toxoplasma infection at least twice during pregnancy (at the beginning and before delivery) for proper management as well as when miscarriage or still birth occurs. Also, the high prevalence of recent or on-going infections necessitates a more comprehensive study 1) to determine the mother-to-child transmission rate of toxoplasmosis in pregnancy; and 2) to determine the role of toxoplasmosis in spontaneous abortions and stillbirths in Ghana. In addition, it is recommended that such studies include other possible infection risk factors that were not considered in this study with the appropriate study design and sample size, to determine the common sources of Toxoplasma transmission. This will help provide substantive data that could contribute to policy formulation towards the institution of appropriate preventive and control measures especially in women of child-bearing age.

\section{REFERENCES}

1. Galván-Ramirez ML, Guillen-Vargas C, SaavedraDuran R, Islos Rodriguez A. Analysis of Toxoplasma gondii antigens with sera from toxoplasmosis patients. Rev Soc Brasil Med Trop 1998; 31: 271-7

2. Thulliez P, Daffos F, Forrestier F. Diagnosis of Toxoplasma infection in the pregnant woman and the unborn child: Current Problems. Scand J Infect Dis 1992; 84; 22-8.

3. Holliman RE. Congenital toxoplasmosis: prevention, screening and treatment. J Hosp Infect 1995; 30: 179-90.

4. McAuley J, Boye KM, Patel D, Beckman J, Schey W, Stein L, Wolters C, Johnson D, Meier P, Mack DG, Mets M, Boyer K, Swisher C, Withers S, Holfels E, McLeod R. . Early and longitudinal evaluation of treated infants and children and untreated historical patients with congenital toxoplasmosis: the Chicago collaborative treatment trial. Clin Infect Dis 1994; 18: 38-72.

5. Guerina N, Hsu H, Meissner C, Maguire J, Lynfield R, Stech-enberg B, et al. Neonatal serologic screening and early treatment for congenital Toxoplasma gondii infection. N Engl J Med 1994; 330(26): 1858-63.

6. Lappin MR, Gasper PW, Rose BJ, Powell CC. Effect of primary phase feline immunodeficiency virus infection on cats with chronic toxoplasmosis. Vet Immunol Immunopathol 1992; 35: 121-31.

7. Al-Hamdani MM, Mahdi NK. Toxoplasmosis among women with habitual abortion. East Med Hlth J. 1997; 3 (2): 310-15.

8. Wallace GD. Experimental transmission of Toxoplasma gondii by filth flies. Am J Trop Med Hyg 1971;.20: 411-13.

9. Montoya JG, Liensenfeld O. Toxoplasmosis. Lanc 2004; 363: 1965-76.

10. Isaac-Renton J, Bowie WR, King A, Irwin GS, Ong CS, Fung CP, Shokeir MO, Dubey JP. Detection of Toxoplasma gondii oocysts in drinking water. Appl. Environ. Microbiol., 1998; 64 ( 6): 2278-80.

11. Bahia-Oliveira LMG, Jones JL, Azevedo-Silva J, Alves CCF, Oréfice F, Addiss DG. High endemic, waterborne toxoplasmosis in north Rio de Janeiro State, Brazil. Emerg Infect Dis 2003; 19: 1-14.

12. Kean BH, Kimball AC, Christenson WN. An epidemic of acute toxoplasmosis. J A M A 1969; 208: 1002-4.

13. Schmidt GD, Roberts LS. Foundations of Parasitology. 6th Ed. 2000.

14. Swisher CN, Boyer K, McLeod R. Congenital Toxoplasmosis. Semin Pediatr Neurol 1994; 1: 425.

15. Sabin AB, Feldman HA. Dyes as micro chemical indicators of a new immunity phenomenon affecting a protozoan parasite (Toxoplasma). Science 1948; 108: 660-3.

16. Montoya JG, Remington JS. Toxoplasmic chorioretinitis in the setting of acute acquired toxoplasmosis. Clin Infect Dis 1996; 23: 277-82.

17. Bessieres MH, Roques C, Berrebi A, Barre V, Cazaux M, Seguela JP. IgA antibody response during acquired and congenital toxoplasmosis. J Clin Pathol 1992; 45: 605-8.

18. Arko-Mensah J, Bosompem KM, Canacoo J, Wastling M, Akanmori BD. The Seroprevalence of toxoplasmosis in pigs in Ghana, Acta Tropica 2000; 76 (1): 27-31.

19. Van der Puije WNA, Bosompem KM, Canacoo EA, Wastling JM, Akanmori BD. The Prevalence of anti-Toxoplasma gondii antibodies in Ghanaian sheep and goats. Acta Tropica 2000; 76(1): 15-20.

20. Anteson RK, Sekimoto S, Furukawa S, Takao Y, Nyanotor MA. Studies on toxoplasmosis in Ghana 
IV. Further evidence of congenital diseases caused by Toxoplasma gondii infections. Gh Med J 1980; 25: 146-8.

21. Ayi I, Akao N, Bosompem KM, Akafo SK, Clarke J, Nyador L, Apea-Kubi K, Fujita K. Development of membrane-bases tests for the detection of urinary antigens and antibodies in human toxoplasmosis: preliminary studies in Ghanaian patients. Acta Tropica 2005; 93: 151-159.

22. Kaaja RJ, Greer IA. Manifestations of chronic disease during pregnancy. J A M A 2005; 294 (21): 2751-57

23. Remington J.S. Toxoplamosis in the adult. Bull NY Acad Med 1974; 50: 211-227.

24. Anteson RK, Sekimoto S, Furukawa S, Quakyi IA. Studies on Toxoplasmosis in Ghana II. The Prevalence of Toxoplasmosis in a group of pregnant women and their neonates. A preliminary report. Gh Med J 1978a; 17: 203-206.

25. Branko Bobič, Ivana Jevremovi ç, Jelena Marinkovi c, Desanka Sibali č and Olgica Djurkovic-Djakovic. Risk factors for Toxoplasma infection in a reproductive age female population in the area of Belgrade, Yugoslavia. European Journal of Epidemiology 1998; 14: 605-610,.
26. Anteson RK, Sekimoto S, Furukawa S, Takao Y, Nyanotor MA. Studies on toxoplasmosis in Ghana I. The prevalence of toxoplasmosis as measured by the haemagglutination (Eiken) test. Ghana Med J 1978b; 17: 147-149.

27. Centers for Diseases Control. http://www.doctoc.com/docs/476518/Toxoplasmaa-Fetal-and-sometimes-Fatal-Parasite 2008

28. Public Health Agency of Canada. http://www.phacaspc.gc.ca/msds-ftss/msds153e-eng.php 2001.

29. Dubey JP, Beattie CP. Toxoplasmosis of animals and man. Boca Raton, Fla: CRC. Press Inc. 1988; 1-220.

30. Dubey JP. Toxoplasmosis. From the Parasite Biology and Epidemiology Laboratory, Livestock and Poultry Sciences Institute, Agricultural Research Service, USDA, Beltsville Agricultural Research Center, Beltsville, MD 1994; 20705-2350. A VM A.

31. Devada K, Anandan R, Dubey JP. Serological prevalence of toxoplasmosis in chickens in Madras. Ind J Parasitol 1998; 84: 621-2.

32. Dubey JP, Thulliez P. Persistence of tissue cysts in edible tissues of cattle fed Toxoplasma gondii oocysts. Am J Vet Res 1993; 54: 270-3. 